\title{
Pacific
}

Journal of

Mathematics

\section{COMMUTANTS OF TOEPLITZ OPERATORS ON THE} BERGMAN SPACE

\author{
ZELJKO CUCKOVIC
}




\section{COMMUTANTS OF TOEPLITZ OPERATORS ON THE BERGMAN SPACE}

\section{ŽELJKO ČUČKOVIĆ}

This paper describes the commutants of certain analytic Toeplitz operators. To underline the difference between the Bergman and Hardy spaces, we first prove that on the Bergman space $L_{a}^{2}$ the only isometric Toeplitz operators with harmonic symbols are scalar multiples of the identity. If $T$ denotes the norm closed subalgebra of $\mathbf{L}\left(L_{a}^{2}\right)$ generated by Toeplitz operators, we show that for each positive integer $n,\left\{T_{z^{n}}\right\}^{\prime} \cap \mathbf{T}$ is the set of all analytic Toeplitz operators. This result is also valid for the Hardy space. Here $\left\{T_{z^{n}}\right\}^{\prime}$ denotes the commutant of $T_{z^{n}}$. Finally we prove the analogous result for $T_{u^{n}}$, where $u$ is an analytic, one-to-one map of the unit disk onto itself.

Introduction. Let $D$ denote the open unit disk in the complex plane and let $d A$ denote the usual Lebesgue area measure on $D$. The complex space $L^{2}(D, d A)$ is a Hilbert space with the inner product

$$
\langle f, g\rangle=\int_{D} f \bar{g} d A .
$$

The Bergman space $L_{a}^{2}$ is the set of those functions in $L^{2}(D, d A)$ that are analytic on $D$. The Bergman space is a closed subspace of $L^{2}(D, d A)$, and so there is an orthogonal projection $P$ from $L^{2}(D, d A)$ onto $L_{a}^{2}$. For $\varphi \in L^{\infty}(D, d A)$, the Toeplitz operator with symbol $\varphi$, denoted $T_{\varphi}$, is the operator from $L_{a}^{2}$ to $L_{a}^{2}$ defined by $T_{\varphi} f=P(\varphi f)$. For more information about the Bergman space and its operators see [4].

The algebra of bounded analytic functions on $D$ will be denoted by $H^{\infty}$. If $\varphi \in H^{\infty}$, then $T_{\varphi}$ is called an analytic Toeplitz operator.

For a Hilbert space $H, \mathbf{L}(H)$ denotes the algebra of all bounded linear operators on $H$. If $S \subset \mathbf{L}(H)$, then $S^{\prime}=\{B \in \mathbf{L}(H): A B=$ $B A$ for all $A \in S\}$ is the commutant of $S$. In this paper we are interested in finding commutants of certain analytic Toeplitz operators acting on the Bergman space.

Much work has been done in studying commutants of analytic Toeplitz operators on the Hardy space. Some of those results can be extended to the Bergman space case. The complex space $L^{2}(\partial D)$ 
is a Hilbert space with the inner product

$$
\langle f, g\rangle=\int_{\partial D} f \bar{g} \frac{d t}{2 \pi} .
$$

For each integer $n$, let $e_{n}$ denote the function $e_{n}(z)=z^{n}$ for $|z|=$ 1. Then $\left\{e_{n}\right\}$ is an orthonormal basis for $L^{2}(\partial D)$ and the Hardy space $H^{2}(\partial D)$ is, by definition, the subspace $\operatorname{span}\left\{e_{n}: n \geq 0\right\}^{-}$. For $\varphi \in L^{\infty}(\partial D)$, the Toeplitz operator with symbol $\varphi$, denoted again by $T_{\varphi}$, on $H^{2}(\partial D)$ is defined in the analogous way. For the basic properties of the Hardy space Toeplitz operators see Douglas [9]. Shields and Wallen [15] studied commutants of certain multiplication operators in a Hilbert space of analytic functions and introduced interesting function theoretic methods. Deddens and Wong [8] studied the problem using operator theory and raised six questions. Abrahamse [1] answered some of Deddens-Wong questions negatively. Baker, Deddens and Ullman [6] found $\left\{T_{f}\right\}^{\prime}$ if $f$ is an entire function. In a series of papers [16]-[19], Thomson used function theoretic methods to find commutants or intersection of commutants of certain analytic Toeplitz operators. Finally, C. Cowen continued their work in [7] and found the commutant of Toeplitz operators whose symbol is a finite Blaschke product or a covering map.

It is well known that the Hardy space Toeplitz operator $T_{\varphi}$ is an isometry if and only if $\varphi$ is inner. If $\varphi$ is a nonconstant inner function, then $T_{\varphi}$ is a pure isometry and is unitarily equivalent to a unilateral shift, whose commutant can be characterized matricially. On the Bergman space, a Toeplitz operator whose symbol is a nonconstant inner function is not an isometry. We will prove even more (Theorem 1.1): The only Toeplitz operator with harmonic symbol that is an isometry is a scalar multiple of the identity.

Our first result about commutants concerns Toeplitz operator with symbol $z^{n}$. Let $\mathbf{T}$ be the norm closed subalgebra of $\mathbf{L}\left(L_{a}^{2}\right)$ generated by all Toeplitz operators. We show (Theorem 1.4) that for each positive integer $n,\left\{T_{z^{n}}\right\}^{\prime} \cap \mathbf{T}$ is the set of all analytic Toeplitz operators. This result is also valid for the Hardy space. Then, we prove the analogous result for $T_{u^{n}}$, where $u$ is an analytic, one-to-one map of $D$ onto itself.

Acknowledgment. This paper represents part of the author's doctoral dissertation written at the Michigan State University under the direction of Professor Sheldon Axler. I am grateful to Professor Sheldon Axler for his guidance and encouragement. 
1. Commutants. At first we will underline the difference between the Bergman and Hardy space Toeplitz operators. We will prove that on the Bergman space there are no nontrivial isometries with harmonic symbols. We need some facts about the maximal ideal space of $H^{\infty}$. Good references are Hoffman [12] and Garnett [10].

The set of all multiplicative linear functionals on $H^{\infty}$ is called the maximal ideal space of $H^{\infty}$ and we denote it by $M$. The Gelfand transform $-: H^{\infty} \rightarrow C(M)$ is defined by $\hat{f}(\varphi)=\varphi(f)$, for $\varphi \in M$. The Gelfand transform is an isometry from $H^{\infty} \rightarrow C(M)$, so that we can identify $H^{\infty}$ with the uniformly closed subalgebra of $C(M)$. Hoffman ([13, Lemma 4.4]) has proved that $C(M)$ is identical to the sup norm closure of the algebra generated by the bounded harmonic functions. If $m_{1}$ and $m_{2}$ are in $M$, the pseudohyperbolic distance between $m_{1}$ and $m_{2}$ is defined as

$$
\rho\left(m_{1}, m_{2}\right)=\sup \left\{\left|\hat{f}\left(m_{2}\right)\right|: f \in H^{\infty},\|f\| \leq 1, \hat{f}\left(m_{1}\right)=0\right\} .
$$

The relation $m_{1} \sim m_{2}$ if and only if $\rho\left(m_{1}, m_{2}\right)<1$ is an equivalence relation on $M$. The corresponding equivalence classes are called the Gleason parts of $M$. The set of one-point parts in $M$ will be denoted by $M_{1}$. Let

$$
J=\left\{\varphi \in C(M): \varphi=0 \text { on } M_{1}\right\} .
$$

Let $\mathbf{T}(C(M))$ be the closed subalgebra of $\mathbf{L}\left(L_{a}^{2}\right)$ generated by $\left\{T_{\varphi}\right.$ : $\varphi \in C(M)\}$ and let $\mathbf{C}$ be the commutator ideal of $\mathbf{T}(C(M))$. McDonald and Sundberg [14] proved that the sequence

$$
0 \rightarrow J \rightarrow C(M) \rightarrow \mathbf{T}(C(M)) / \mathbf{C} \rightarrow 0
$$

is exact. This implies that $C(M) / J$ is isomorphic to $\mathbf{T}(C(M)) / \mathbf{C}$ with isomorphism

$$
\Psi(\varphi+J)=T_{\varphi}+\mathbf{C}
$$

for $\varphi \in C(M)$.

Another Banach algebra we need is $L^{\infty}(\partial D)$. The maximal ideal space of $L^{\infty}(\partial D)$, denoted by $M\left(L^{\infty}\right)$, plays an important role here. Since we may regard $H^{\infty}$ as a closed subalgebra of $L^{\infty}(\partial D)$, we may think of $M\left(L^{\infty}\right)$ as a subset of $M$. It turns out that $M\left(L^{\infty}\right)$ is a subset of $M_{1}$.

Now, we can prove our theorem.

Theorem 1.1. Suppose that $h \in L^{\infty}(D, d A)$ is harmonic and that $T_{h}$ is an isometry. Then $h$ is a constant function of modulus 1. 
Proof. Suppose that $T_{h}$ is an isometry, i.e., $T_{h}^{*} T_{h}=I$. Since $h$ is harmonic, Hoffman's result shows that $h$ and $\bar{h} \in C(M)$. Applying the isomorphism $\Psi^{-1}$, we obtain $(\bar{h}+J) \cdot(h+J)=1+J$, and by the definition of $J$ we have

$$
|\varphi(h)|=1
$$

for every $\varphi \in M\left(L^{\infty}\right)$. It is well known that the Gelfand transform of $L^{\infty}(\partial D)$ maps $L^{\infty}(\partial D)$ isometrically and isomorphically onto $C\left(M\left(L^{\infty}\right)\right.$ ) (see Hoffman, [12, p. 170]), so that we have

$$
\begin{aligned}
\sup \{|h(z)|: z \in D\} & =\|h\|_{L^{\infty}(\partial D)}=\|\hat{h}\|_{C\left(M\left(L^{\infty}\right)\right)} \\
& =\sup \left\{|\varphi(h)|: \varphi \in M\left(L^{\infty}\right)\right\} .
\end{aligned}
$$

Hence (1.1) implies $\sup \{|h(z)|: z \in D\}=1$. If $|h(z)|=1$ for some $z \in D$, then $h$ is constant by the Maximum Principle. If $|h(z)|<1$ for all $z \in D$, then

$$
\pi=\|1\|^{2}=\left\|T_{h} 1\right\|^{2}=\|P h\|^{2} \leq\|h\|^{2}=\int_{D}|h(z)|^{2} d A<\int_{D} d A=\pi,
$$

a contradiction. Here $\|\cdot\|$ denotes the $L^{2}(D, d A)$-norm. Therefore $h$ is a constant function, and since $T_{h}$ is an isometry, $|h|=1$.

We can slightly generalize this result and get the following:

CoRollary 1.2. Suppose that $T_{h}$ is an isometry, where $h=f \cdot g^{n}$, where $f$ is inner, $g \in L^{\infty}(D, d A)$ is harmonic, and $n \in \mathbf{N}$. Then $h$ is a constant function of modulus 1 .

J. Thukral asked for which harmonic $h$ is $T_{h}$ a partial isometry. If $T_{h}$ is a partial isometry, then by Halmos [11, Problem 98], $T_{h}=$ $T_{h} T_{h}^{*} T_{h}$. Similarly as before, this means

$$
\varphi(h)\left[1-|\varphi(h)|^{2}\right]=0
$$

for every $\varphi \in M\left(L^{\infty}\right)$. If $\varphi(h)=0$ for every $\varphi \in M\left(L^{\infty}\right)$, then $h=0$. If $\varphi(h) \neq 0$ for some $\varphi \in M\left(L^{\infty}\right)$, then $\sup \{|h(z)|: z \in$ $D\}=1$ and $h$ must be a constant function. Thus we have proved the following theorem:

Theorem 1.3. Suppose that $h \in L^{\infty}(D, d A)$ is harmonic and that $T_{h}$ is a partial isometry. Then $h$ is either a constant function of modulus 1 or $h$ is identically 0.

In [5] S. Axler and the author characterized commuting Toeplitz operators with harmonic symbols. Now, we are going to consider the 
related problem - the commutants of some analytic Toeplitz operators. At first we will be interested in finding the commutant of $T_{z^{n}}$, for arbitrary positive integer $n$. Before we state and prove our result, recall the following definitions.

For $\varphi \in L^{\infty}(D, d A)$, the Hankel operator with symbol $\varphi$, denoted $H_{\varphi}$, is the operator from $L_{a}^{2}$ to $\left(L_{a}^{2}\right)^{\perp}$ defined by $H_{\varphi} f=(I-P)(\varphi f)$.

For an analytic function $f$ on $D$ we set

$$
\|f\|_{B}=\sup \left\{\left(1-|z|^{2}\right)\left|f^{\prime}(z)\right|: z \in D\right\} .
$$

The Bloch space $B$ is the set of all analytic functions $f$ on $D$ for which $\|f\|_{B}<\infty$. The quantity $|f(0)|+\|f\|_{B}$ defines a norm on $B$, and $B$ equipped with this norm is a Banach space. Contained in the Bloch space is the little Bloch space $B_{0}$, which is by definition the set of all analytic functions $f$ on $D$ for which

$$
\left(1-|z|^{2}\right) f^{\prime}(z) \rightarrow 0 \quad \text { as }|z| \rightarrow 1 \text {. }
$$

For the basic properties of the Bloch space see [2].

Let $n \in \mathbf{N}$ be fixed.

TheOREM 1.4. Let $S \in \mathbf{T}$ commute with $T_{z^{n}}$. Then $S=T_{\psi}$ for some $\psi \in H^{\infty}$.

Proof. The equation $S T_{z^{n}}=T_{z^{n}} S$ gives us the following:

Let $g_{i}=S z^{i}$, for $i=0,1, \ldots, n-1$. Then for any such $i$

$$
\begin{aligned}
& S z^{n+i}=S T_{z^{n}} z^{i}=T_{z^{n}} S z^{i}=z^{n} g_{i} \\
& \ldots \\
& S z^{k n+i}=z^{k n} g_{i}, \quad \text { for } k=0,1,2 \ldots
\end{aligned}
$$

Let

$$
\begin{aligned}
& X_{0}=\operatorname{span}\left\{e_{k n}: k=0,1,2, \ldots\right\}^{-}, \\
& X_{1}=\operatorname{span}\left\{e_{k n+1}: k=0,1,2, \ldots\right\}^{-}, \\
& \ldots \\
& X_{n-1}=\operatorname{span}\left\{e_{k n+(n-1)}: k=0,1,2, \ldots\right\}^{-} .
\end{aligned}
$$

Then $L_{a}^{2}=X_{0} \oplus X_{1} \oplus \cdots \oplus X_{n-1}$, i.e., each $f \in L_{a}^{2}$ can be written as

$$
f=f_{0}+f_{1}+\cdots+f_{n-1},
$$

$f_{i} \in X_{i}, i=0,1,2, \ldots, n-1$. Each $f_{0} \in X_{0}$ has its Fourier series expansion

$$
f_{0}=\sum_{k=0}^{\infty}\left(f_{0}, e_{k n}\right) e_{k n}
$$


i.e., $f_{0}=\lim s_{m}$, where $s_{m}=\sum_{k=0}^{m}\left(f_{0}, e_{k n}\right) e_{k n}$. Since the point evaluations are bounded on $L_{a}^{2}$, we have $f_{0}(z)=\lim s_{m}(z)$, for each $z \in D$, so that

$$
\left(f_{0} \cdot g_{0}\right)(z)=\lim \left(s_{m} \cdot g_{0}\right)(z)
$$

for each $z \in D$. Since $S z^{k n}=z^{k n} g_{0}, k=0,1,2, \ldots$, it follows that $S s_{m}=s_{m} \cdot g_{0}$, for every $m \in \mathbf{N}$. By continuity of $S$, we have

$$
\left(S f_{0}\right)=\lim S s_{m}=\lim s_{m} \cdot g_{0},
$$

so that

$$
\left(S f_{0}\right)(z)=\lim \left(s_{m} \cdot g_{0}\right)(z)
$$

for each $z \in D$. Comparing (1.2) and (1.3) we conclude that

$$
S f_{0}=g_{0} \cdot f_{0},
$$

for each $f_{0} \in X_{0}$. Repeating the above reasoning, we get that $S f_{1}=$ $g_{1} f_{1} / z$, for $f_{1} \in X_{1}$ and so on. Thus the operator $S$ can be described as

$$
S f=g_{0} f_{0}+\frac{g_{1}}{z} f_{1}+\frac{g_{2}}{z^{2}} f_{2}+\cdots+\frac{g_{n-1}}{z^{n-1}} f_{n-1} .
$$

Let's observe another property of $S$, being an element of $\mathbf{T}$.

Claim. $S T_{z}-T_{z} S \in \mathbf{K}$, where $\mathbf{K}$ denotes the ideal of all compact operators.

At first, assume $S=T_{\varphi}, \varphi \in L^{\infty}(D, d A)$. Then

$$
T_{\varphi} T_{z}-T_{z} T_{\varphi}=T_{z \varphi}-T_{z} T_{\varphi}=H_{\bar{z}}^{*} H_{\varphi} .
$$

Because $z \in B_{0}$, the operator $H_{\bar{z}}^{*}$ is compact (see [3]), so that $T_{\varphi} T_{z}$ $T_{z} T_{\varphi}$ is compact. If $\Pi: \mathbf{L}\left(L_{a}^{2}\right) \rightarrow \mathbf{L}\left(L_{a}^{2}\right) / \mathbf{K}$ denotes the natural projection, then

$$
\Pi\left(T_{\varphi}\right) \Pi\left(T_{z}\right)=\Pi\left(T_{z}\right) \Pi\left(T_{\varphi}\right)
$$

for every $\varphi \in L^{\infty}(D, d A)$. If $S=T_{\varphi_{1}} \cdots T_{\varphi_{l}}$ then, because of (1.5), $\Pi\left(S T_{z}-T_{z} S\right)=0$ so that $S T_{z}-T_{z} S \in \mathbf{K}$. An arbitrary operator $S$ in $\mathbf{T}$ is the limit of sums of operators of the form $T_{\varphi_{1}} \cdots T_{\varphi_{1}}$. In that case $S=\lim S_{l}$, each $S_{l}$ is of the form $\sum T_{\varphi_{1}} \cdots T_{\varphi_{l}}$, and so $S T_{z}-T_{z} S=\lim \left(S_{l} T_{z}-T_{z} S_{l}\right) \in \mathbf{K}$. Hence the claim is proved.

Now, let's express $S T_{z}-T_{z} S$ in terms of (1.4). It is easy to see that

$$
S T_{z} f=g_{1} f_{0}+\frac{g_{2}}{z} f_{1}+\frac{g_{3}}{z^{2}} f_{2}+\cdots+z g_{0} f_{n-1} .
$$


From this and (1.4) we obtain

$$
\begin{aligned}
\left(S T_{z}-T_{z} S\right) f= & f_{0}\left(g_{1}-z g_{0}\right)+f_{1}\left(\frac{g_{2}}{z}-g_{1}\right) \\
& +f_{2}\left(\frac{g_{3}}{z^{2}}-\frac{g_{2}}{z}\right)+\cdots+f_{n-1}\left(z g_{0}-\frac{g_{n-1}}{z^{n-2}}\right) .
\end{aligned}
$$

By the claim, $\left.\left(S T_{z}-T_{z} S\right)\right|_{X_{0}}=M_{g_{1}-z g_{0}}: X_{0} \rightarrow L_{a}^{2}$ is compact $\left(M_{g_{1}-z g_{0}}\right.$ is a multiplication operator). Let $\varphi=g_{1}-z g_{0}$. Now, it immediately follows that $\left.M_{\varphi}\right|_{X}$ is compact, for all $j=1, \ldots, n-1$, since $\left.M_{\varphi}\right|_{X_{j}}=M_{z^{j}}\left(\left.M_{\varphi}\right|_{X_{0}}\right)\left(\left.M_{z^{-j}}\right|_{X_{j}}\right)$. Hence $M_{\varphi}$ is compact on $X_{0} \oplus X_{1} \oplus \cdots \oplus X_{n-1}=L_{a}^{2}$. Thus $\varphi=0$ and therefore $g_{0}=g_{1} / z$.

Similarly, $\left.\left(S T_{z}-T_{z} S\right)\right|_{X_{1}}=M_{g_{2} / z-g_{1}}: X_{1} \rightarrow L_{a}^{2}$ is a compact operator. Thus $\left.M_{g_{2} / z-g_{1}} M_{z}\right|_{X_{0}}=M_{g_{2}-z g_{1}}: X_{0} \rightarrow L_{a}^{2}$ is compact. As before, this means that $g_{2}-z g_{1}=0$ so that $g_{1}=g_{2} / z$ and therefore $g_{0}=g_{2} / z^{2}$. If we continue this way, (1.4) shows that

$$
S f=g_{0} \cdot f=T_{g_{0}} f
$$

for every $f \in L_{a}^{2}$. The function $g_{0}$ must be an $H^{\infty}$ function as a multiplier of $L_{a}^{2}$ (see [15]). If we let $\psi=g_{0}$, the theorem is proved.

REMARK. From the proof of the theorem it is clear that we can replace the assumption $S \in \mathbf{T}$ by the weaker assumption $S T_{z}-T_{z} S \in$ K. Also, slightly modified arguments give a proof for the Hardy space case.

We can extend this result. Let $\operatorname{Aut}(D)$ denote the set of analytic, one-to-one maps of $D$ onto $D$. Let $u \in \operatorname{Aut}(D)$ and define an operator $V: L_{a}^{2} \rightarrow L_{a}^{2}$ by $V f=f \circ u^{-1}$. Clearly, $V$ is a bounded linear operator, with the inverse operator $V^{-1} f=f \circ u$. Observe that

$$
T_{z} V=V T_{u}
$$

and therefore

$$
T_{z^{n}} V=V T_{u^{n}}
$$

for every $n \in \mathbf{N}$.

Suppose now that $S \in \mathbf{T}$ and $S T_{u^{n}}=T_{u^{n}} S$ for some $n$. Formula (1.6) implies

$$
S V^{-1} T_{z^{n}} V=V^{-1} T_{z^{n}} V S
$$

and we conclude that

$$
V S V^{-1} \in\left\{T_{z^{n}}\right\}^{\prime}
$$


The operator $B=V S V^{-1}$ has the property that $B T_{z}-T_{z} B$ is in $\mathbf{K}$ (because $u \in B_{0}$ ), so by the remark following Theorem 1.4, it follows that $B=T_{\varphi}$, for some $\varphi \in H^{\infty}$. Thus $S=V^{-1} T_{\varphi} V$. If we let $\psi=\varphi \circ u$, we have proved the following corollary:

Corollary 1.5. Let $u \in \operatorname{Aut}(D)$ and let $S \in \mathbf{T}$ commute with $T_{u^{n}}$, for some $n \in \mathbf{N}$. Then $S=T_{\psi}$ for some $\psi \in H^{\infty}$.

The results in this paper raise the following questions.

The McDonald-Sundberg functional calculus is the crucial tool in proving Theorem 1.1. Is this theorem true without the assumption on the symbol to be harmonic? If $h$ is in $C(M)$, then the McDonaldSundberg calculus is still valid, but it is not true in general that $\sup \{|h(z)|: z \in D\}=\sup \left\{|\varphi(h)|: \varphi \in M\left(L^{\infty}\right)\right\}$. However, we guess that there are no nontrivial isometries among Toeplitz operators on the Bergman space.

Suppose $S \in \mathbf{L}\left(L_{a}^{2}\right)$ is such that $S T_{z}-T_{z} S \in \mathbf{K}$. If $S T_{z^{n}}-T_{z^{n}} S=0$ for some $n$, then Theorem 1.4 shows that $S$ must be an analytic Toeplitz operator. What is the set of all functions $f$ such that $S T_{f}-$ $T_{f} S=0$ implies that $S$ is an analytic Toeplitz operator?

\section{REFERENCES}

[1] M. B. Abrahamse, Analytic Toeplitz operators with automorphic symbol, Proc. Amer. Math. Soc., 52 (1975), 297-302.

[2] J. M. Anderson, Bloch functions: The Basic theory, Operators and Function Theory, Reidel Publishing Company, Dordrecht, 1985, pp. 1-17.

[3] S. Axler, The Bergman space, the Bloch space, and commutators of multiplication operators, Duke Math. J., 53 (1986), 315-332.

[4] _ Bergman spaces and their operators, Surveys of Some Recent Results in Operator Theory, Vol. 1, Longman, 1988, pp. 1-50.

[5] S. Axler and Ž. Čučković, Commuting Toeplitz operators with harmonic symbols, Integral Equations Operator Theory, 14 (1991), 1-12.

[6] I. N. Baker, J. A. Deddens and J. L. Ullman, $A$ theorem on entire functions with applications to Toeplitz operators, Duke Math. J., 41 (1974), 739-745.

[7] C. C. Cowen, The commutant of an analytic Toeplitz operator, Trans. Amer. Math. Soc., 239 (1978), 1-31.

[8] J. A. Deddens and T. K. Wong, The commutant of analytic Toeplitz operators, Trans. Amer. Math. Soc., 184 (1973), 261-273.

[9] R. G. Douglas, Banach Algebra Techniques in Operator Theory, Academic Press, New York, 1972.

[10] J. B. Garnett, Bounded Analytic Functions, Academic Press, New York, 1981.

[11] P. R. Halmos, Hilbert Space Problem Book, Springer-Verlag, New York, 1974.

[12] K. Hoffman, Banach Spaces of Analytic Functions, Dover, New York, 1962.

[13] _ Bounded analytic functions and Gleason parts, Ann. of Math., 86 (1967), 74-111. 
[14] G. McDonald and C. Sundberg, Toeplitz operators on the disc, Indiana Univ. Math. J., 28 (1979), 595-611.

[15] A. L. Shields and L. J. Wallen, The commutants of certain Hilbert space operators, Indiana Univ. Math. J., 20 (1970/71), 777-788.

[16] J. E. Thomson, Intersections of commutants of analytic Toeplitz operators, Proc. Amer. Math. Soc., 52 (1975), 305-310.

[17] - The commutants of certain analytic Toeplitz operators, Proc. Amer. Math. Soc., 54 (1976), 165-169.

[18] _ The commutant of a class of analytic Toeplitz operators, Amer. J. Math., 99 (1977), 522-529.

[19] - The commutant of a class of analytic Toeplitz operators II, Indiana Univ. Math. J., 25 (1976), 793-800.

Received April 17, 1992 and in revised form October 23, 1992.

UNIVERSITY OF WISCONSIN CENTERS

WAUKESHA, WI 53188 



\title{
PACIFIC JOURNAL OF MATHEMATICS
}

\author{
Founded by
}

\author{
E. F. BECKENBACH (1906-1982) F. Wolf (1904-1989)
}

\section{EDITORS}

Sun-Yung A. Chang

(Managing Editor)

University of California

Los Angeles, CA 90024-1555

chang@math.ucla.edu

\section{F. Michael Christ}

University of California

Los Angeles, CA 90024-1555

christ@math.ucla.edu

Herbert Clemens

University of Utah

Salt Lake City, UT 84112

clemens@math.utah.edu
THOMAS ENRIGHT

University of California, San Diego

La Jolla, CA 92093

tenright@ucsd.edu

Nicholas ERCOLANI

University of Arizona

Tucson, AZ 85721

ercolani@math.arizona.edu

R. FINN

Stanford University

Stanford, CA 94305

finn@gauss.stanford.edu

VAUGHAN F. R. JONES

University of California

Berkeley, CA 94720

vfr@math.berkeley.edu
SteVen KerCKHofF

Stanford University

Stanford, CA 94305

spk@gauss.stanford.edu

MARTIN ScharLemanN

University of California

Santa Barbara, CA 93106

mgscharl@math.ucsb.edu

Harold STARK

University of California, San Diego

La Jolla, CA 92093

V. S. VARADARAJAN

University of California

Los Angeles, CA 90024-1555

vsv@math.ucla.edu

\section{SUPPORTING INSTITUTIONS}

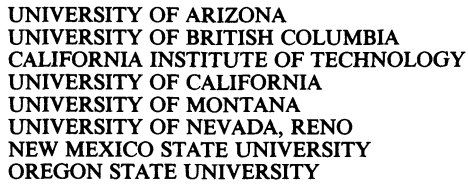

The Supporting Institutions listed above contribute to the cost of publication of this Journal, but they are not owners or publishers and have no responsibility for its content or policies.

Mathematical papers intended for publication in the Pacific Journal of Mathematics should be in typed form or offset-reproduced (not dittoed), double spaced with large margins. Please do not use built up fractions in the text of the manuscript. However, you may use them in the displayed equations. Underline Greek letters in red, German in green, and script in blue. The first paragraph must be capable of being used separately as a synopsis of the entire paper. In particular it should contain no bibliographic references. Please propose a heading for the odd numbered pages of less than 35 characters. Manuscripts, in triplicate, may be sent to any one of the editors. Please classify according to the 1991 Mathematics Subject Classification scheme which can be found in the December index volumes of Mathematical Reviews. Supply name and address of author to whom proofs should be sent. All other communications should be addressed to the managing editor, or Julie Honig, University of California, Los Angeles, California 90024-1555.

There are page-charges associated with articles appearing in the Pacific Journal of Mathematics. These charges are expected to be paid by the author's University, Government Agency or Company. If the author or authors do not have access to such Institutional support these charges are waived. Single authors will receive 75 free reprints; joint authors will receive a total of 100 free reprints. Additional copies may be obtained at cost in multiples of 50 .

The Pacific Journal of Mathematics (ISSN 0030-8730) is published monthly except for July and August. Regular subscription rate: $\$ 215.00$ a year (10 issues). Special rate: $\$ 107.50$ a year to individual members of supporting institutions.

Subscriptions, orders for numbers issued in the last three calendar years, and changes of address should be sent to Pacific Journal of Mathematics, P.O. Box 4163, Berkeley, CA 94704-0163, U.S.A. Old back numbers obtainable from Kraus Periodicals Co., Route 100, Millwood, NY 10546.

The Pacific Journal of Mathematics at University of California, c/o Department of Mathematics, 981 Evans Hall, Berkeley, CA 94720 (ISSN 0030-8730) is published monthly except for July and August. Second-class postage paid at Berkeley, CA 94704, and additional mailing offices. POSTMASTER: send address changes to Pacific Journal of Mathematics, P.O. Box 4163, Berkeley, CA 94704-0163.

PUBLISHED BY PACIFIC JOURNAL OF MATHEMATICS at University of California, Berkeley, CA 94720, A NON-PROFIT CORPORATION

This publication was typeset using $\mathcal{A} \mathcal{M} S-\mathrm{T}_{\mathrm{E}} \mathrm{X}$, the American Mathematical Society's $\mathrm{T}_{\mathrm{E}} \mathrm{X}$ macro system. Copyright (c) 1994 by Pacific Journal of Mathematics 


\section{PACIFIC JOURNAL OF MATHEMATICS}

Volume $162 \quad$ No. $2 \quad$ February 1994

On the existence of convex classical solutions to multilayer fluid

201 problems in arbitrary space dimensions

ANDREW FRENCH ACKER

Extremal functions and the Chang-Marshall inequality

VALENTIN V. ANDREEV and ALEC LANE MATHESON

Productive polynomials

RICHARD ARENS

On factor representations of discrete rational nilpotent groups and the 261 Plancherel formula

LAWRENCE JAY CORWIN and CAROLYN PFEFFER JOHNSTON

Commutants of Toeplitz operators on the Bergman space

ZELJKO CUCKOVIC

When $L^{1}$ of a vector measure is an AL-space

GUILlERMO P. CURBERA

A convexity theorem for semisimple symmetric spaces

305

KARL-HERMANN NEEB

Ideals of finite codimension in free algebras and the fc-localization

AMNON RosenmanN and ShMUEL Rosset

Dec groups for arbitrarily high exponents

BHARATH Al SETHURAMAN

Errata to: "The set of primes dividing the Lucas numbers has density $2 / 3$ "

JEFFREY C. LAGARIAS 